\title{
Novel explanation for the shape of the lenticular galaxy bulge and its implication for red spiral galaxy evolution
}

\author{
R. A. Schachar ${ }^{1}$, G. G. Liao $^{2}$, R. D. Kirby ${ }^{2}$, F. Kamangar ${ }^{3}$, Z. E. Musielak ${ }^{1,4}$, and G. Rosensteel ${ }^{5}$ \\ 1 Department of Physics, University of Texas at Arlington, Arlington, TX 76019, USA \\ e-mail: ron@2ras.com \\ 2 Department of Mathematics, University of Texas at Arlington, Arlington, TX 76019, USA \\ 3 Department of Computer Science and Engineering, University of Texas at Arlington, Arlington, TX 76019, USA \\ 4 Kiepenheuer-Institut für Sonnenphysik, Schöneckstr. 6, Freiburg, 79104 Germany \\ 5 Department of Physics, Tulane University, New Orleans, LA 70118, USA
}

Received 28 March 2009 / Accepted 28 July 2009

\section{ABSTRACT}

\begin{abstract}
Aims. According to Hubble's classification scheme, lenticular galaxies represent an intermediate evolutionary step between elliptical and spiral galaxies. This evolutionary path predicts that the aspect ratios of both lenticular and spiral galaxies should be smaller than the aspect ratios of their E6 or E7 elliptical predecessors. In contradiction to this prediction, observation has demonstrated that the aspect ratio of lenticular galaxies is larger than its immediate elliptical predecessor. In this paper, we suggest a novel explanation for this inconsistency.

Methods. The approach described in this paper is primarily based on analytical methods; however, some numerical methods are also used.

Results. Our idea comes from theoretical and experimental results, which show that a small increase in the equatorial diameter of an oblate spheroid with an aspect ratio $\leq 0.6$ surprisingly causes its minor axis to also increase. We demonstrate that the same phenomenon occurs in the isodensity contours of elliptical galaxies given by Miyamoto \& Nagai (Miyamoto M., \& Nagai R., 1975, PASJ, 27, 533) and in a Maclaurin spheroidal mass in response to the gravitational force generated by a circumferential equatorial disk.

Conclusions. The result of this paper is our explanation for the transformation of a disky elliptical galaxy into a lenticular galaxy which in response to rotation and equatorial diameter expansion evolves into a red spiral galaxy. This evolutionary path is consistent with the common environmental location of disky ellipticals, lenticular and red spiral galaxies and explains why elliptical galaxies are generally $\leq \mathrm{E} 4$. The proposed evolutionary path is opposite to the generally accepted formation of lenticular galaxies from the merger of spiral galaxies.
\end{abstract}

Key words. galaxies: bulge - galaxies: elliptical, lenticular, cD - galaxies: evolution

\section{Introduction}

Lenticular galaxies, labeled S0 in Hubble's classification scheme, form a transition between elliptical and spiral galaxies (Hubble 1926, 1936). The fact that this transition is smooth, which means that some S0 galaxies might well be classified as ellipticals and others as spirals, has led to controversy as to whether lenticular galaxies are more closely linked to elliptical or spiral galaxies (van den Bergh 1980). Jeans (1928) postulated that lenticular galaxies evolve from elliptical galaxies as a consequence of rotation (Sandage 2005). Consistent with Jeans' postulate, Miyamoto \& Nagai (1975) showed that a family of decreasing isodensity contours with declining aspect ratios can describe the morphological differences of elliptical and spiral galaxies (see also Satoh 1980, Zhenglu 2000, Baes 2008). However, there are two basic problems with this evolutionary path:

First, spiral galaxies are bluer in color, signifying that they are younger in age than the redder elliptical and lenticular galaxies. Recently, Bamford et al. (2009) studied the morphology, color, environment and stellar mass of galaxies using data from over $10^{5}$ objects from Galaxy Zoo, the largest sample of visually classified morphologies yet compiled. Interestingly, they demonstrate the existence of red spiral galaxies located in the same environment as elliptical and lenticular galaxies and show that there is an environmental dependence for color beyond that for morphology. This finding implies that the evolution of spiral galaxies may depend on their environment. Young blue spiral galaxies are routinely observed in less dense environments where there are fewer lenticular galaxies. Red spirals are located in denser environments where there are many elliptical and lenticular galaxies. Does this imply different evolutionary paths for young and old spiral galaxies?

Second, it has been observed that the mean aspect ratio, minor axis/major axis ratio, of lenticular galaxies is $0.5 \pm 0.25$ (calculated from Table 2 of Michard \& Marchal 1994), which is greater than E6 and E7 elliptical galaxies, that have aspect ratios of 0.4 and 0.3 , respectively. According to the Hubble Jean's scheme E6 and/or E7 galaxies should evolve by rotation into lenticular galaxies. But how can this occur when the aspect ratio and therefore the minor axis of lenticular galaxies is greater than their immediate elliptical predecessors?

The latter question is addressed by the general belief that lenticular galaxies form as a consequence of the collision of spiral galaxies (Abraham \& van den Bergh 2001, Bedregal et al. 2006); however, this hypothesis has not yet been confirmed by N-body simulations (Hernandez \& Lee 2004, Maller et al.2006). In addition, the complex relationship between morphology, 


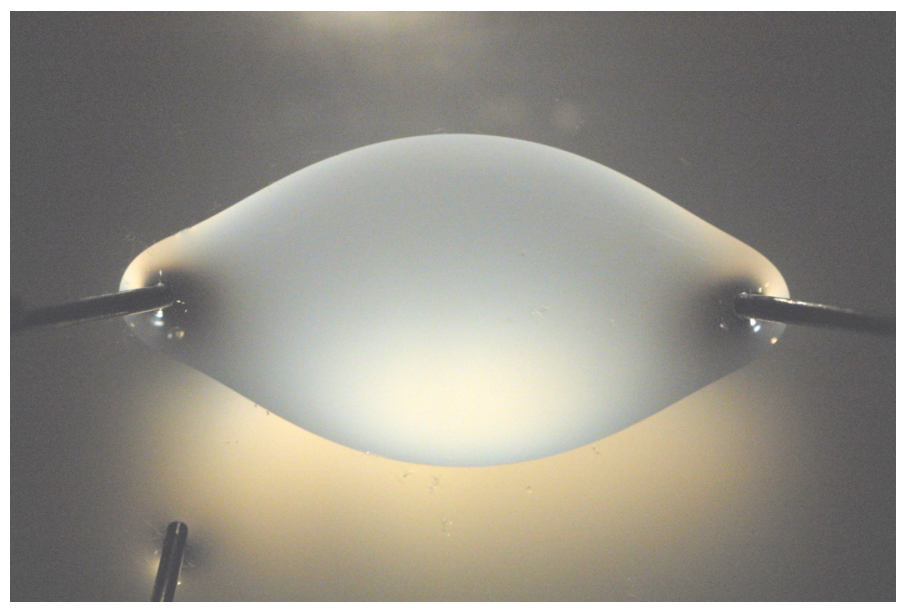

Fig. 1. Photograph of a "steep profile" induced by applying traction in the 180 degree meridian to an oil film floating on water. The tractional pins extend into the oil film and not into the water below.

color, stellar mass and the environmental location of galaxies implies a gentle evolutionary path, not violent major mergers (Bamford et al.2009).

The purpose of this paper is to suggest, consistent with Bamford's et al. (2009) data, that there may be separate pathways for the development of blue (younger) and red (older) spiral galaxies. This differentiation is based on our explanation for the increase of the minor axis of an elliptical galaxy as it transforms into a lenticular galaxy. The idea is based on the change in shape that occurs when the major axis of an encapsulated biconvex object with a long oval cross sectional profile is increased $\leq 5 \%$, counterintuitively, the length of its minor axis also increases, its central surfaces steepen, and its peripheral surfaces flatten; i.e., the object morphologically obtains a "steep profile". It has been demonstrated that this morphological "steep profile" occurs as a consequence of minimization of surface curvature, a surrogate for bending energy (Schachar et al. 2008). The morphological "steep profile" has a remarkable resemblance to the edge-on isophotal shape of a lenticular galaxy bulge as shown by the "steep profile" induced in a floating oil film in response to equatorial traction (Fig. 1). The floating oil film experiment is shown as an example of the effect of minimization of energy on object shape and not to imply that the hydrodynamics, molecular forces and surface tension of the floating oil film are directly applicable to a rotating body of stars controlled by gravity.

To demonstrate that the same phenomenon can be important for galaxy evolution, we consider the shape changes that occur to isodensity contours of elliptical galaxies given by Miyamoto \& Nagai (1975) and a Maclaurin spheroidal mass in response to the gravitational force of a circumferential equatorial disk. In both cases, similar morphological "steep profiles" are observed. The obtained results are used to justify our suggestion that disky elliptical galaxies transform into lenticular galaxies, which then evolve into red spiral galaxies as a consequence of an increase in the equatorial diameter of the lenticular galaxy (see Sects. 2 and 3). Our discussion and conclusions can be found in Sects. 4 and 5. Obviously, our goal is not to develop a detailed model of spiral galaxy evolution, but only to demonstrate that the phenomenon may be relevant.

\section{Transformation of galaxies with Miyamoto \& Nagai's isodensity contours into "steep profiles"}

When a circumferential equatorial disk is added to an elliptical galaxy, the disk applies a gravitational force that expands the galaxy in its equatorial plane. The redistribution of the galaxy's mass changes the overall shape of the galaxy so that it is in a minimal energy state. To determine the new shape of the galaxy, the minimal energy state of the mass distribution of the galaxy is simulated by minimizing the curvature of the galaxy's isodensity contour.

Using solutions of Poisson's equation, Miyamoto \& Nagai (1975) calculated the isodensity contours of a rotating elliptical galaxy with declining density distribution. They show that the induced density change can account for the morphological differences between elliptical and spiral galaxies; however, their analysis does not predict the existence of a lenticular galaxy between an elliptical and spiral galaxy with an aspect ratio greater than both types of galaxies.

It has been well established that most elliptical galaxies have disks (Bender et al. 1989. Rix \& White 1990, Jørgensen \& Franx 1994, Magorrian 1999. Cappellari 2008, Krajnović et al. 2008) and these disky elliptical galaxies are distinguishable from lenticular galaxies (Jaffe et al. 1994, Caon \& Einasto 1995. Katsiyannis et al.2001, Kormendy et al. 2009). We propose that the gravitational force generated by the circumferential equatorial disk increases the equatorial diameter (major axis) of the elliptical galaxy causing it to evolve into a lenticular galaxy. To assess this possibility, we evaluate the morphological changes that will occur to an elliptical galaxy when its major axis is increased.

We begin by calculating the shape of an elliptical galaxy using cylindrical coordinates as given by Miyamoto \& Nagai's (1975)

$\rho(R, z)=\frac{b^{2} M}{4 \pi} \frac{a R^{2}+\left[q+3 \sqrt{z^{2}+b^{2}}\right]\left[a+\sqrt{z^{2}+b^{2}}\right]}{\left\{R^{2}+\left[a+\sqrt{z^{2}+b^{2}}\right]^{2}\right\}^{\frac{5}{2}}\left(z^{2}+b^{2}\right)^{\frac{3}{2}}}$

where $\rho$ is density, $M$ is total mass, and $a$ and $b$ are positive constants with dimensions of length. We modify this equation by introducing dimensionless vertical and horizontal coordinates, $Z=\frac{z}{a}$ and $X=\frac{R}{a}$, respectively, and letting $q=\frac{b}{a}$ so that

$\rho(X, Z)=\frac{q^{2} M}{4 \pi a^{3}} \frac{X^{2}+\left[1+3 \sqrt{Z^{2}+q^{2}}\right]\left[1+\sqrt{Z^{2}+q^{2}}\right]^{2}}{\left.\left\{X^{2}+\left[1+\sqrt{Z^{2}+q^{2}}\right]^{2}\right)\right\}^{\frac{5}{2}}\left(Z^{2}+q^{2}\right)^{\frac{3}{2}}}$,

where $\rho, M, a^{3}$ and $4 \pi$ are incorporated into a single parameter, $P=\frac{4 \pi \rho a^{3}}{M}$ and then

$P(X, Z)=\frac{q^{2}\left\{X^{2}+\left[1+3 \sqrt{Z^{2}+q^{2}}\right]\left[1+\sqrt{Z^{2}+q^{2}}\right]^{2}\right\}}{\left.\left\{X^{2}+\left[1+\sqrt{Z^{2}+q^{2}}\right]^{2}\right)\right\}^{\frac{5}{2}}\left(Z^{2}+q^{2}\right)^{\frac{3}{2}}}$

Since the mean aspect ratio of lenticular galaxies is 0.5 and the Jeans-Hubble scheme predicts an E6 or E7 elliptical galaxy will convert into a lenticular galaxy, shape parameters are selected to cover this range of elliptical galaxies; i.e., E7 to E4 $(0.3 \leq$ aspect ratio $\leq 0.6)$. For each elliptical galaxy the density parameter, $P$, is varied from 0.1 to 0.0001 .

The curvature of the isodensity contour is given by

$\kappa(X, Z)=\frac{\left|Z^{\prime \prime}\right|}{\left(1+Z^{\prime 2}\right)^{\frac{3}{2}}}$. 
To simulate the gravitational effect of a circumferential equatorial disk, the major axis of the isodensity contour of the elliptical galaxy is increased $1.5 \%$. To ensure the new galaxy shape is in the lowest energy state, the calculus of variation is used to minimize the square of the difference of the curvatures between the isodensity contour of the elliptical galaxy and the new galaxy contour

$$
\int\left[\kappa(Z(X))-\kappa\left(Z_{\mathrm{o}}(X)\right)\right]^{2} \mathrm{~d} s .
$$

The problem is solved numerically using standard optimization software with the following constraints:

Arc length

$$
\int \sqrt{1+Z_{\mathrm{o}}^{\prime 2}} \mathrm{~d} X \leq \int \sqrt{1+Z^{\prime 2}} \mathrm{~d} X \leq n \int \sqrt{1+Z_{\mathrm{o}}^{\prime 2}} \mathrm{~d} X .
$$

Area

$$
\int Z_{\mathrm{o}} \mathrm{d} X \leq \int Z \mathrm{~d} X \leq n \int Z_{\mathrm{o}} \mathrm{d} X
$$

Surface area

$$
\begin{aligned}
\int X \sqrt{1+Z_{\mathrm{o}}^{\prime 2}} \mathrm{~d} X & \leq \int X \sqrt{1+Z^{\prime 2}} \mathrm{~d} X \\
& \leq n \int X \sqrt{1+Z_{\mathrm{o}}^{\prime 2}} \mathrm{~d} X
\end{aligned}
$$

where $1.01 \leq n \leq 1.03$ and volume is unconstrained.

The boundary conditions are

$$
\begin{aligned}
Z\left(1.015 X_{\mathrm{o}}\right) & =0, \\
\left.\frac{\mathrm{d} X}{\mathrm{~d} Z}\right|_{Z=0} & =0, \\
\left.\frac{\mathrm{d} Z}{\mathrm{~d} X}\right|_{X=0} & =0,
\end{aligned}
$$

where $\left(X_{0}, 0\right)$ is the intercept of the isodensity contour with the major axis of the elliptical galaxy.

The obtained results are given in Figs. 24, which show that in each case the minor axis of the galaxy isodensity contour increases as a consequence of an increase in its major axis transforming the galaxy isodensity contour into a "steep profile".

\section{Formation of a "steep profile" in a Maclaurin spheroid with a toroidal disk}

The shape changes of a Maclaurin spheroidal mass in response to increasing angular momentum have been used to predict the shapes of galaxies (Sandage 2005). With increasing angular momentum the Maclaurin spheroidal mass becomes more oblate until it has an eccentricity of 0.8 , which is equivalent to an aspect ratio of 0.6. At this point, the Maclaurin spheroid is unstable and with a decrease in angular momentum will become a Jacobi ellipsoid (Eriguchi \& Hachisu 1982, Dankova \& Rosensteel 1998). Alternatively, we propose that if a Maclaurin spheroid mass has an aspect ratio $\leq 0.6$ and has a circumferential equatorial disk, the Maclaurin spheroid will morphologically form a "steep profile" while angular momentum remains constant.
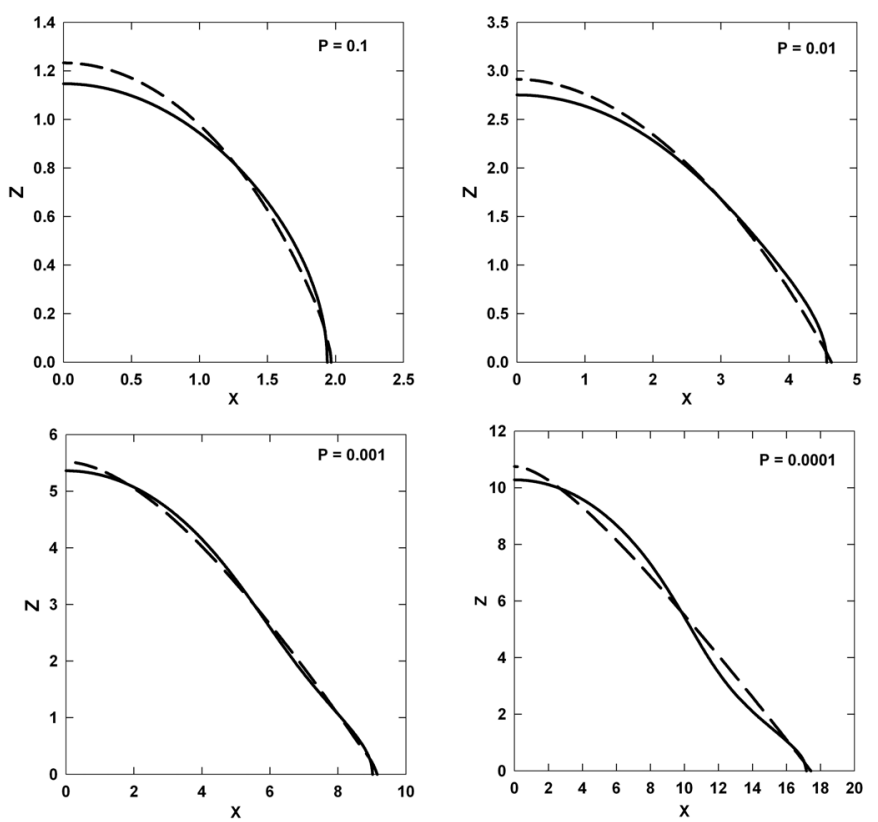

Fig. 2. Simulation of the effect of a circumferential equatorial disc on the shape of galaxy isodensity contours (solid curve) with aspect ratios $=0.6$ and densities $P=0.1-0.0001$, which all change into "steep profiles" (dashed curves).
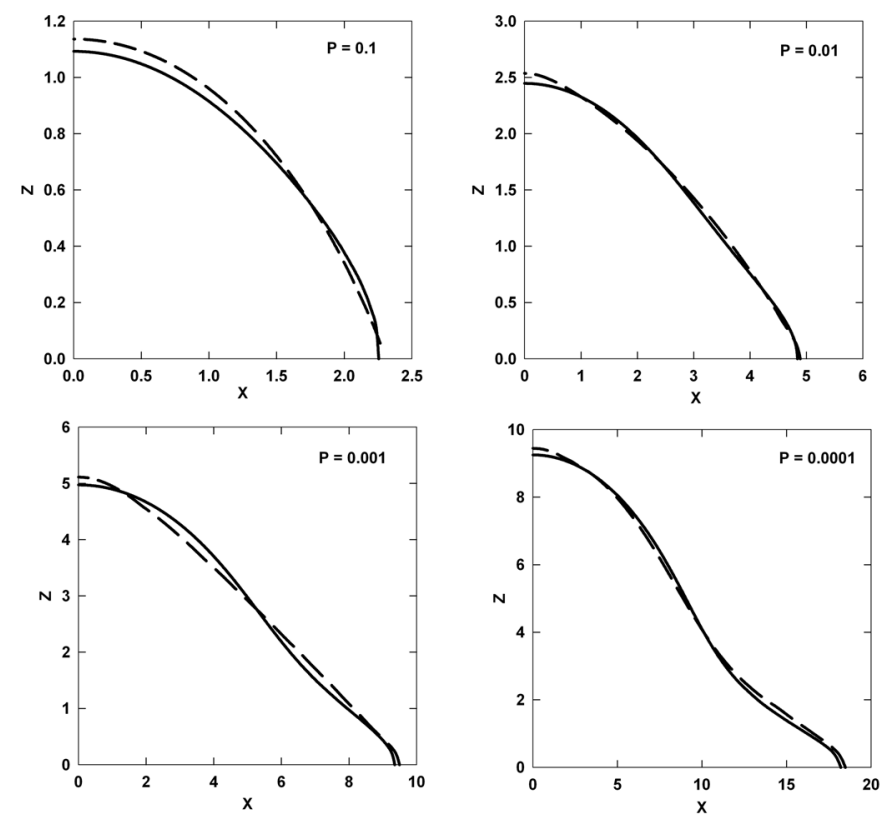

Fig. 3. Simulation of the effect of a circumferential equatorial disc on the shape of galaxy isodensity contours (solid curve) with aspect ratios $=0.5$ and densities $P=0.1-0.0001$, which all change into "steep profiles" (dashed curves).

\subsection{Configuration}

Consider the symmetry axis of a surface of revolution aligned with the $z$-axis. The surface bounds a connected region $\Omega$ that contains a total mass $M$ in a volume $V=\operatorname{vol}(\Omega)$. The mass is distributed uniformly inside the surface. Using cylindrical coordinates, the profile of the surface is defined by a function $r=f(z)$ for $-z_{m} \leq z \leq z_{m}$. For each point $z$ along the symmetry axis inside the surface, the polar coordinate ranges from $r=0$, the symmetry axis, to $r=f(z)$, the boundary of the surface. The surface of revolution is symmetrical: $f(-z)=f(z)$ for $-z_{m} \leq z \leq z_{m}$. 

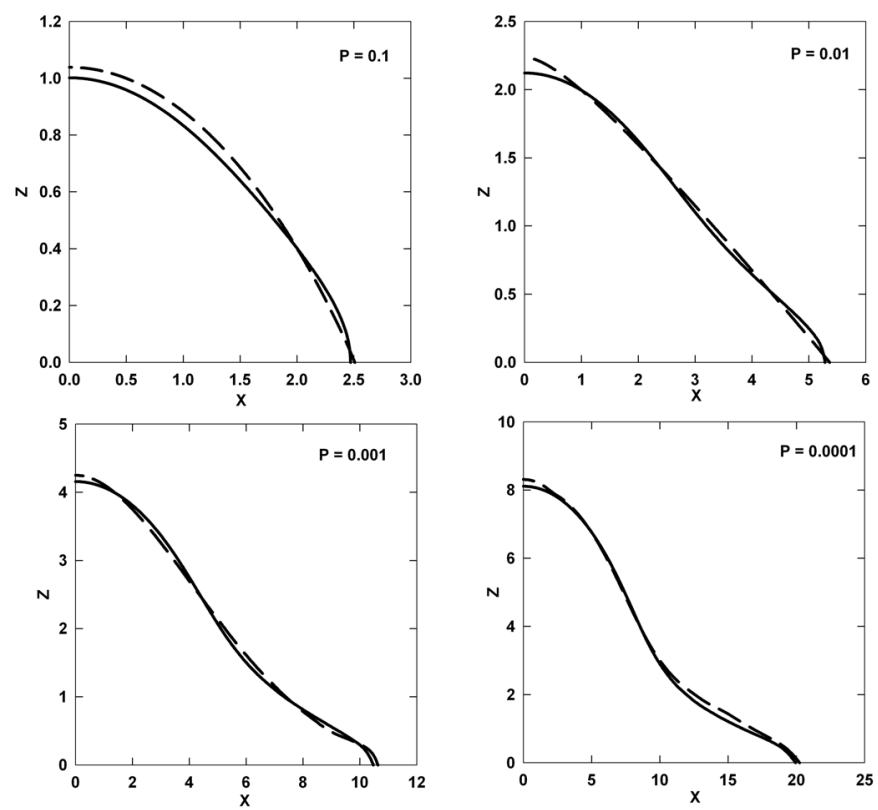

Fig. 4. Simulation of the effect of a circumferential equatorial disc on the shape of galaxy isodensity contours (solid curve) with aspect ratios $=0.4$ and densities $P=0.1-0.0001$, which all change into "steep profiles" (dashed curves).
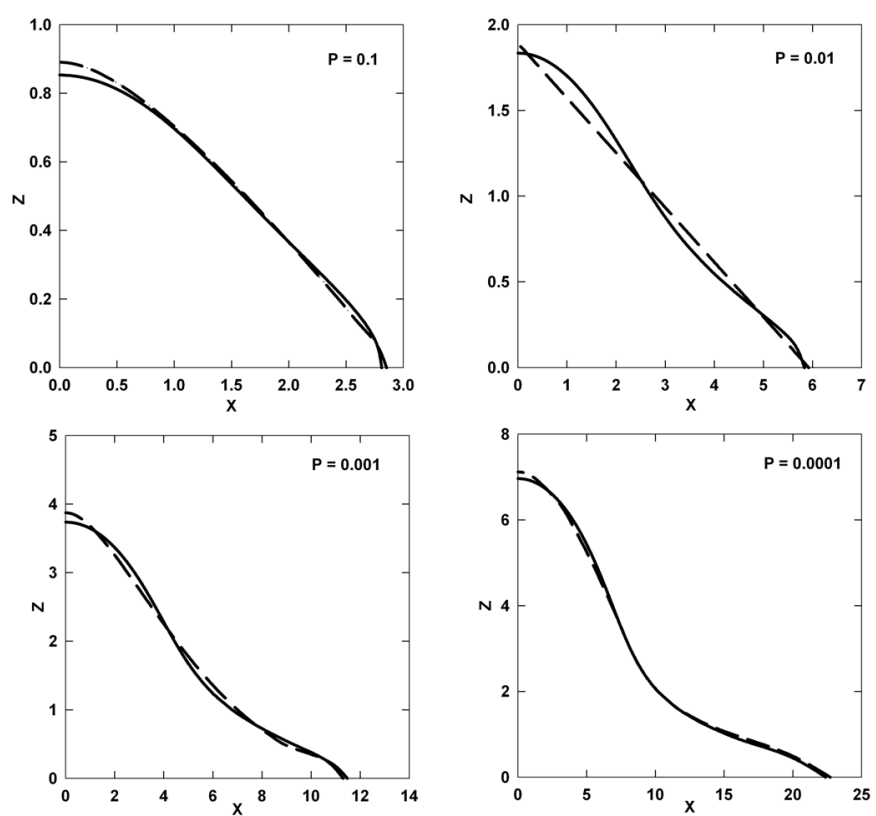

Fig. 5. Simulation of the effect of a circumferential equatorial disc on the shape of galaxy isodensity contours (solid curve) with aspect ratios $=0.3$ and densities $P=0.1-0.0001$, which all change into "steep profiles" (dashed curves).

Another mass $M_{\text {ext }}$ is distributed uniformly along the circumference of a circle of radius $R$ in the $z=0$ plane. This circle is centered at the origin, which is the geometrical center of the symmetrical surface. The radius $R>>f(0)>z_{m}>0$. The external mass models a toroidal galactic disk.

The total potential energy $U$ is the sum of two terms: 1) the gravitational interaction energy $U_{\text {ext }}$ between the mass $M$ and the external mass $M_{\text {ext }}$ and 2) the gravitational self-energy $U_{\text {self }}$ of the mass. The total kinetic energy is just the kinetic energy of the

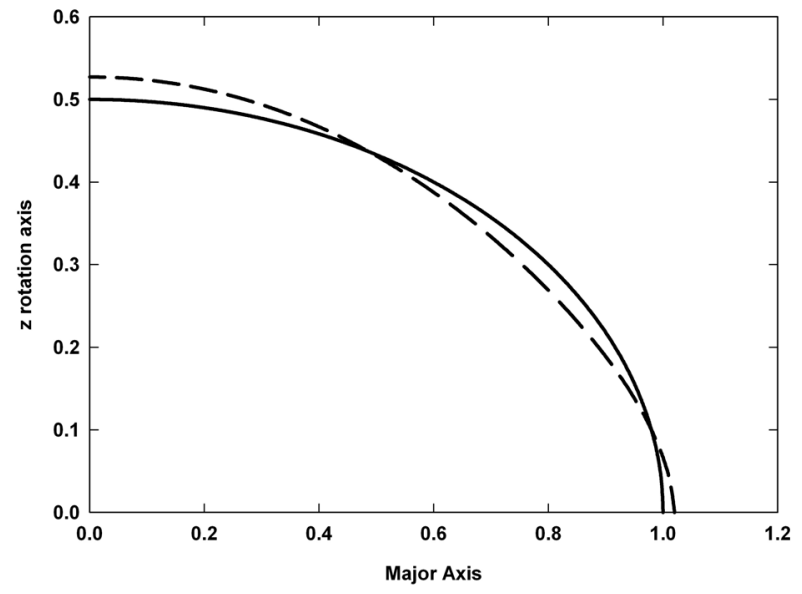

Fig. 6. Reference ellipse (solid curve) and "steep profile" (dashed curve).

rigidly rotating mass $M$ within the region $\Omega$. The kinetic energy and self-energy of the toroidal mass are considered constants.

\subsection{Volume of the Maclaurin spheroid}

To determine the volume of the mass, imagine the mass is divided into narrow cylindrical slices perpendicular to the symmetry axis. The slice at position $z$ along the axis has width $\mathrm{d} z$ and radius $r=f(z)$. The slice's volume is $\pi|f(z)|^{2} \mathrm{~d} z$ and the mass's volume is the integral

$V=\int_{-z_{m}}^{z_{m}} \pi|f(z)|^{2} \mathrm{~d} z=2 \pi \int_{0}^{z_{m}}|f(z)|^{2} \mathrm{~d} z$

For an oblate spheroid, the profile is $f_{\mathrm{sph}}(z)=a \sqrt{1-z^{2} / c^{2}}$, where $c=z_{m}$ denotes the minor semi-axis length and $a>c$ is the major semi-axis length. The volume is $V=4 \pi a^{2} c / 3$.

\subsection{Two-dimensional "steep profile" and volume corrections}

Using a reference ellipse with a semimajor axis length $a=1$ (arbitrary units) and semiminor axis length $c=1 / 2$ and the "steep profile" generated by increasing the major axis of the reference ellipse $2 \%$, the difference $\Delta f$ between the "steep profile's" function, $r=f(z)$, and the ellipse's function, $r=f_{\mathrm{sph}}(z)$, are plotted in Fig. 6.

The difference function is approximated by three segments (Fig.7).

$r-r_{\mathrm{sph}}=\left\{\begin{array}{l}\Delta f_{1}=0.0208-0.1593 z-0.5336 z^{2} \\ \Delta f_{2}=(0.1527 z-0.0664) /(1-1.9056 z), \\ \Delta f_{3}=1.61(0.528-z)^{0.493}\end{array}\right.$

where $\Delta f_{1}, \Delta f_{2}$ and $\Delta f_{3}$ are valid in the range $0 \leq z \leq 0.253$, $0.253 \leq z \leq 0.50$ and $0.50 \leq z \leq 0.528$, respectively.

The area of one quadrant of the ellipse is $\pi a c / 4=\pi / 8=$ 0.393 . The difference between the single quadrant areas bounded by the "steep profile" and ellipse is

$$
\begin{gathered}
\int_{0}^{z_{m}} f(z) \mathrm{d} z-\int_{0}^{c} f_{\mathrm{sph}}(z) \mathrm{d} z=\int_{0}^{0.253} \Delta f_{1} \mathrm{~d} z \\
+\int_{0.253}^{0.5} \Delta f_{2} \mathrm{~d} z+\int_{0.5}^{0.528} \Delta f_{3} \mathrm{~d} z=-0.000067 .
\end{gathered}
$$




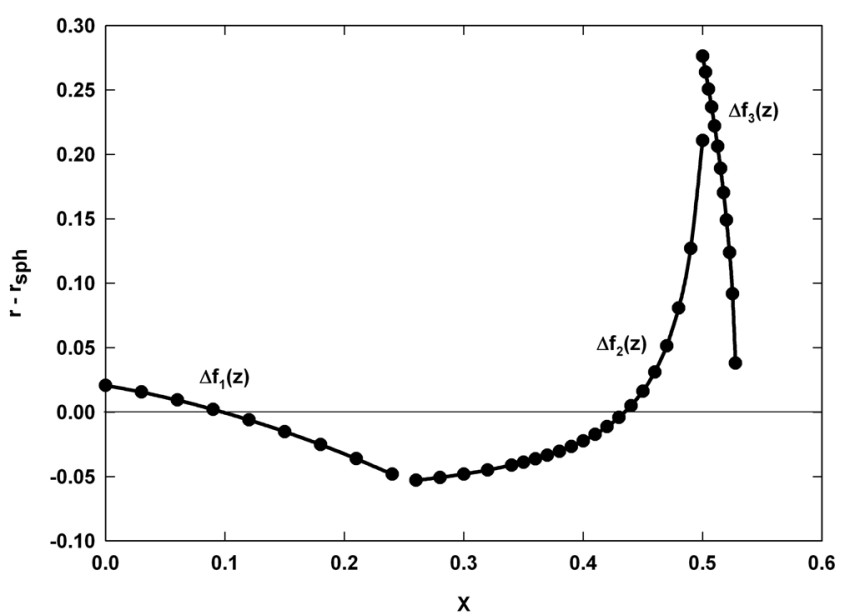

Fig. 7. Difference function $r-r_{\text {sph }}$ between the "steep profile" curve and the reference ellipse, which is approximated by three segments.

The fractional change in the quadrant's area is $0.02 \%$, a negligible shift.

\subsubsection{Three-dimensional surface of revolution}

The difference between the volume of the "steep profile" and the volume of the ellipsoid is

$\Delta V=2 \pi \int_{0}^{z_{m}}|f(z)|^{2} \mathrm{~d} z-2 \pi \int_{0}^{c}\left|f_{\mathrm{sph}}(z)\right|^{2} \mathrm{~d} z$

The difference $\Delta g$ between the square of the "steep profile's" function and the square of the ellipse's function is approximated by three segments (Fig. 8)

$\Delta g_{1}(z)=0.0379-4.4286 z^{2}+72.7588 z^{4}-554.5930 z^{6}$,

$\Delta g_{2}(z)=-0.0637-0.6697 z^{2}+6.8028 z^{4}-7.5373 z^{6}$,

$\Delta g_{3}(z)=0.7366-2.6411 z^{2}$,

where $z \in I_{1}=[0,0.2392)$ for $\Delta g_{1}(z), z \in I_{2}=[0.2392,0.50)$ for $\Delta g_{2}(z)$, and $z \in I_{3}=[0.50,0.5281]$ for $\Delta g_{3}(z)$.

The function $\Delta g$ is

$\Delta g(z)=\Delta g_{1}(z) \chi_{I_{1}}(z)+\Delta g_{2}(z) \chi_{I_{2}}(z)+\Delta g_{3}(z) \chi_{I_{3}}(z)$,

where the characteristic function is

$\chi_{I}(z)=\left\{\begin{array}{l}1, z \in I \\ 0, z \notin I\end{array}\right.$

The volume of the ellipsoid is $4 \pi a^{2} c / 3=2 \pi / 3=2.094$. The difference between the volumes bounded by the "steep profile" and ellipse is

$$
\begin{aligned}
\Delta V= & 2 \pi \int_{0}^{0.239} \Delta g_{1} \mathrm{~d} z+2 \pi \int_{0.239}^{0.5} \Delta g_{2} \mathrm{~d} z \\
& +2 \pi \int_{0.5}^{0.528} \Delta g_{3} \mathrm{~d} z=-0.06650 .
\end{aligned}
$$

The fractional change in the volume is $-3.2 \%$.

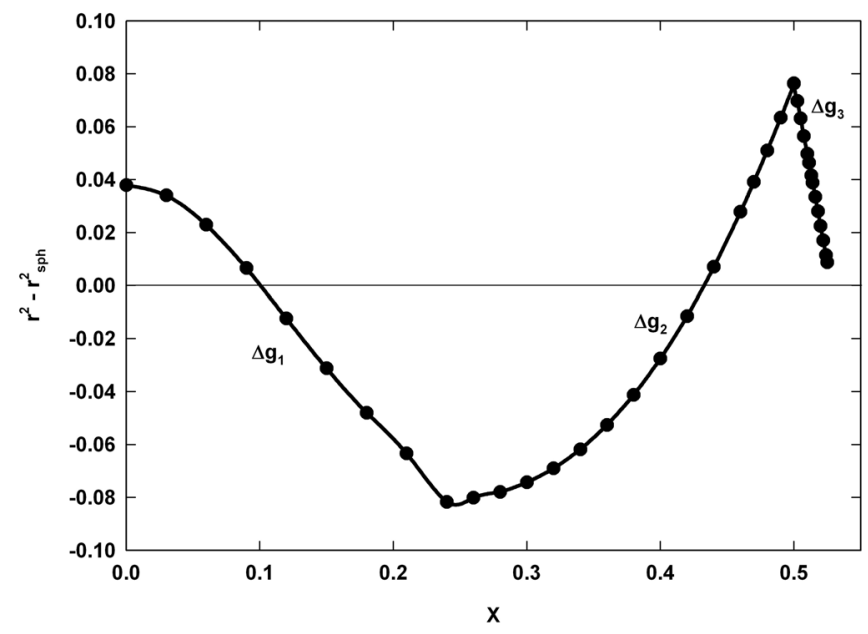

Fig. 8. Difference $\Delta g$ between the square of the "steep profile's" function and the square of the ellipse's function, which is approximated by three segments.

\subsubsection{Volume correction}

The volumes $V_{\epsilon}$ of the surfaces of revolution, indexed by $\epsilon>0$ and defined by the cylindrical radial function

$r_{\epsilon}^{2}(z)=r_{\mathrm{sph}}^{2}(z)+\epsilon \Delta g(z)$

where the ellipsoid's radial function is

$r_{\mathrm{sph}}^{2}(z)=\left(1-4 z^{2}\right) \chi_{I_{\mathrm{sph}}}(z), I_{\mathrm{sph}}=[0,0.5]$,

are less than the volume of the reference spheroid $V_{\mathrm{sph}}=2 \pi / 3$ :

$\frac{V_{\epsilon}}{V_{\mathrm{sph}}}=1-0.03175 \epsilon$.

To reset the volumes equal to the ellipsoid's value, define the dilation

$r_{\epsilon \lambda}(z)=\lambda r_{\epsilon}(z / \lambda)$

for $\lambda \geq 1$. The volume of the new surface of revolution is $V_{\epsilon \lambda}=$ $\lambda^{3} V_{\epsilon}$ which is set equal to $V_{\text {sph }}$ (Fig. 9). Therefore, the volumecorrecting factor $\lambda$ is

$\lambda=(1-0.03175 \epsilon)^{-1 / 3}=1+0.01058 \epsilon+\cdots$

\subsection{Kinetic energy of the Maclaurin spheroid and the "steep profile"}

When the surface of revolution rotates rigidly about its symmetry $z$-axis, the moment of inertia is

$\mathcal{I}=\int \mathrm{d} z \int 2 \pi r \mathrm{~d} r \cdot \mathrm{d} r^{2}=\pi d \int_{0}^{\infty} \mathrm{d} z|r(z)|^{4}$,

where $d=M / V$ is the uniform density. When the surface is a spheroid with semi-axis lengths, $a=b$ and $c$, the moment of inertia is

$\mathcal{I}_{\mathrm{sph}}=\mathcal{I}_{0}(a / R)^{2}=\mathcal{I}_{0}\left(1-e^{2}\right)^{-1 / 3}, \quad \mathcal{I}_{0}=\frac{2}{5} M R^{2}$, 


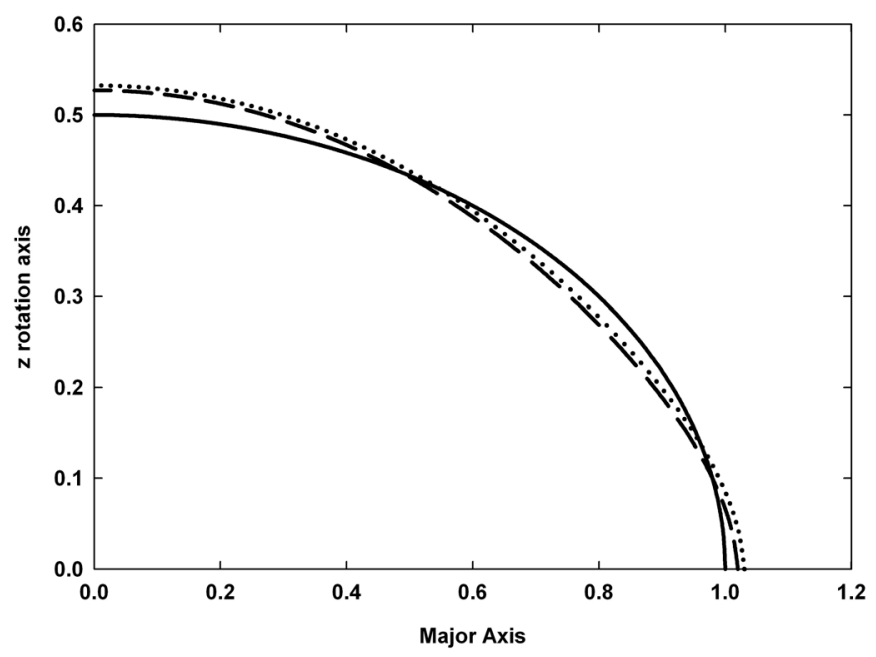

Fig. 9. Ellipse (solid curve), "steep profile" (dashed curve) and volumecorrected "steep profile" (dotted curve).

where $R^{3}=a^{2} c$ and the eccentricity is $e=\sqrt{1-c^{2} / a^{2}}$. The ellipsoid has semi-axis lengths $a=b=1$ and $c=1 / 2$, eccentricity $e=\sqrt{3} / 2, R=2^{-1 / 3}, \mathcal{I}_{0}=\frac{2}{5} M 4^{-1 / 3}$, and $\mathcal{I}_{\mathrm{sph}}=\frac{2}{5} M$. is

For the volume-corrected surface of revolution, the moment

$$
\begin{aligned}
\mathcal{I} & =\pi \mathrm{d} \lambda^{4} \int_{0}^{\infty} \mathrm{d} z\left|r_{\epsilon}(z / \lambda)\right|^{4}=\pi \mathrm{d} \lambda^{5} \int_{0}^{\infty} \mathrm{d} u\left|r_{\epsilon}(u)\right|^{4} \\
& =\pi \mathrm{d} \lambda^{5} \int_{0}^{\infty} \mathrm{d} u\left|r_{\mathrm{sph}}^{2}(u)+\epsilon \Delta g(u)\right|^{2} \\
& \approx \pi \mathrm{d} \lambda^{5} \int_{0}^{\infty} \mathrm{d} u\left[r_{\mathrm{sph}}^{4}(u)+2 \epsilon r_{\mathrm{sph}}^{2}(u) \Delta g(u)\right]
\end{aligned}
$$

When $\epsilon=0$, the moment of inertia simplifies to the ellipsoidal value

$$
\begin{aligned}
\mathcal{I}_{\mathrm{sph}} & =\pi \mathrm{d} \int_{0}^{\infty} \mathrm{d} z r_{\mathrm{sph}}^{4}(z) \\
& =\pi \mathrm{d} \int_{0}^{1 / 2}\left(1-4 z^{2}\right)^{2} \mathrm{~d} z=\frac{2 M}{5} .
\end{aligned}
$$
file" is

The moment of inertia of the volume-corrected "steep pro-

$\mathcal{I}_{\epsilon \lambda}=\mathcal{I}_{\mathrm{sph}}(1-0.01234 \epsilon)$.

The kinetic energy is the square of the angular momentum $L$ divided by twice the moment of inertia. Let $U_{0}$ denote a convenient energy unit (chosen below to give the gravitational selfenergy of a sphere of mass $M$ and radius $R$ ) and define the dimensionless angular momentum $\lambda=L / \sqrt{I_{0} U_{0}}$. The kinetic energy, in units of $U_{0}$, of a spheroid is

$T_{\mathrm{sph}}=\frac{L^{2}}{2 I_{\mathrm{sph}} U_{0}}=\frac{1}{2} \lambda^{2}\left(1-e^{2}\right)^{1 / 3}$,

and for the "steep profile"

$T_{\epsilon \lambda}=T_{\mathrm{sph}}(1+0.01234 \epsilon)$.

\subsection{Self-energy of the "steep profile"}

The "steep profile" forms as a result of a small incompressible deformation of the spheroid. The mass density $d(\boldsymbol{r})$ equals a constant $d$ for a position vector $\boldsymbol{r}$ inside the "steep profile" and equals zero for $\boldsymbol{r}$ outside the region $\Omega$. The mass density of the ellipsoid, $d_{\mathrm{sph}}(\boldsymbol{r})$, equals $d$ when $\boldsymbol{r}$ is inside the ellipsoid and equals zero for $\boldsymbol{r}$ outside the ellipsoid. The difference between these densities is a function $\delta d(\boldsymbol{r})=d(\boldsymbol{r})-d_{\mathrm{sph}}(\boldsymbol{r})$ which is zero except for a narrow domain around the surface of the ellipsoid. More generally, consider a family of "steep profiles" indexed by a real number $\epsilon$ with the densities

$d_{\epsilon}(\boldsymbol{r})=d_{\mathrm{sph}}(\boldsymbol{r})+\epsilon \delta d(\boldsymbol{r})$.

At $\epsilon=0$ the "steep profile's" density $d_{\epsilon}$ equals the ellipsoid's density $d_{\text {sph }}$. The self-energy of a "steep profile" is

$U_{\text {self }}=-\frac{G}{2} \iint \frac{d_{\epsilon}(\boldsymbol{r}) d^{3} r d_{\epsilon}\left(\boldsymbol{r}^{\prime}\right) d^{3} r^{\prime}}{\left|\boldsymbol{r}-\boldsymbol{r}^{\prime}\right|}$,

where $G$ is the universal gravitational constant and $\left|\boldsymbol{r}-\boldsymbol{r}^{\prime}\right|$ denotes the distance between positions $\boldsymbol{r}$ and $\boldsymbol{r}^{\prime}$. To order $\epsilon$ the self-energy is

$$
\begin{aligned}
U_{\mathrm{self}} \approx & -\frac{G}{2} \iint \frac{d_{\mathrm{sph}}(\boldsymbol{r}) d^{3} r d_{\mathrm{sph}}\left(\boldsymbol{r}^{\prime}\right) d^{3} r^{\prime}}{\left|\boldsymbol{r}-\boldsymbol{r}^{\prime}\right|} \\
& -\epsilon G \iint \frac{d_{\mathrm{sph}}\left(\boldsymbol{r}^{\prime}\right) d^{3} r^{\prime} \delta d(\boldsymbol{r}) d^{3} r}{\left|\boldsymbol{r}-\boldsymbol{r}^{\prime}\right|} .
\end{aligned}
$$

The first term is the Maclaurin spheroid's self-energy $U_{\text {sph }}$ and is a known quantity (Chandrasekhar 1969. Dankova \& Rosensteel 1998,

$U_{\mathrm{sph}}=-U_{0}\left(1-e^{2}\right)^{1 / 6} \frac{\arcsin (e)}{e}$,

where the eccentricity $e=\sqrt{1-c^{2} / a^{2}}$. The self-energy $-U_{0}$ of a reference sphere of radius $R$ and mass $M$ is determined by $U_{0}=(3 / 5) G M^{2} / R$, where $R^{3}=a^{2} c$.

The second integral may be expressed in terms of the function

$U_{\text {surf }}(\boldsymbol{r})=-G \int \frac{d_{\mathrm{sph}}\left(\boldsymbol{r}^{\prime}\right) d^{3} r^{\prime}}{\left|\boldsymbol{r}-\boldsymbol{r}^{\prime}\right|}$.

Therefore, the "steep profile's" self-energy is, to order $\epsilon$,

$U_{\text {self }}=U_{\text {sph }}+\epsilon \int \delta d(\boldsymbol{r}) U_{\text {surf }}(\boldsymbol{r}) d^{3} r$.

Since $\delta d(\boldsymbol{r})$ is zero outside of a narrow region near the ellipsoid's surface, the potential energy $U_{\text {surf }}(\boldsymbol{r})$ due to the ellpsoidal mass must be determined only for points $\boldsymbol{r}$ near the ellipsoid's surface.

For the "steep profile's" surface of revolution, the integral is

$$
\begin{aligned}
U_{\text {self }}-U_{\text {sph }} & =d \int \mathrm{d} z \int_{r_{\mathrm{sph}}(z)}^{r_{\epsilon \lambda}(z)} 2 \pi r \mathrm{~d} r U_{\mathrm{surf}}(\boldsymbol{r}) \\
& \approx \pi d \int \mathrm{d} z\left(r_{\epsilon \lambda}^{2}(z)-r_{\mathrm{sph}}^{2}(z)\right) U_{\text {surf }}(z),
\end{aligned}
$$

where, to obtain overall accuracy of order $\epsilon$, it is sufficient to evaluate the potential energy $U_{\text {surf }}(z)$ at the surface, Eq. (38). The potential energy at the surface is the sum of two terms, a constant 
term and a term proportional to $z^{2}$. The integral involving the constant term is zero due to volume conservation.

Let us define $\Delta U \equiv U_{\text {self }}-U_{\text {sph }}$ and write

$$
\begin{aligned}
\Delta U & =\pi d \int \mathrm{d} z\left(r_{\epsilon \lambda}^{2}-r_{\mathrm{sph}}^{2}\right)(-\pi G d)\left(A_{1} \frac{a^{2}}{c^{2}}-A_{3}\right) z^{2} \\
& =-(\pi d)^{2} G\left(A_{1} \frac{a^{2}}{c^{2}}-A_{3}\right) \int \mathrm{d} z\left(\lambda^{2} r_{\epsilon}^{2}-r_{\mathrm{sph}}^{2}\right) z^{2} .
\end{aligned}
$$

The first term in this integral, to order $\epsilon$, is

$$
\begin{aligned}
\int \mathrm{d} z \lambda^{2} r_{\epsilon}^{2}\left(\frac{z}{\lambda}\right) z^{2}= & \lambda^{5} \int \mathrm{d} u\left(r_{\mathrm{sph}}^{2}(u)+\epsilon \Delta g(u)\right) u^{2} \\
= & \int \mathrm{d} u r_{\mathrm{sph}}^{2}(u) u^{2}+\epsilon \int \mathrm{d} u\left(0.0529 r_{\mathrm{sph}}^{2}(u)\right. \\
& +\Delta g(u)) u^{2},
\end{aligned}
$$

since $\lambda^{5} \approx 1+0.0529 \epsilon$. Therefore, to order $\epsilon$, the change in the self-energy is

$$
\begin{aligned}
\Delta U & =-(\pi d)^{2} G\left(A_{1} \frac{a^{2}}{c^{2}}-A_{3}\right) \epsilon(0.0529 / 30-0.000922) \\
& =-(\pi d)^{2} G(0.000704) \epsilon \\
& =-(0.002095) U_{0} \epsilon,
\end{aligned}
$$

where $A_{1} \frac{a^{2}}{c^{2}}-A_{3}=0.8368$.

\subsection{Potential energy of the Maclaurin spheroid}

The surface of the ellipsoid is defined by the equation, $1=$ $x_{1}^{2} / a_{1}^{2}+x_{2}^{2} / a_{2}^{2}+x_{3}^{2} / a_{3}^{2}$. The gravitational potential energy for the interior and exterior of a homogeneous ellipsoid with semiaxis lengths $a_{1}, a_{2}, a_{3}$ is defined by two quantities: the gravitational potential energy $\mathfrak{V}$ and the gravitational self-energy $\mathfrak{W}$ (Chandrasekhar 1969).

\subsubsection{Interior points}

At an interior point $\boldsymbol{r}=\left(x_{1}, x_{2}, x_{3}\right)$, the potential energy (Chandrasekhar 1969) is

$$
\mathfrak{V}=\pi G d\left(I-\sum_{\ell=1}^{3} A_{\ell} x_{\ell}^{2}\right) \text {, }
$$

where $I=\sum a_{\ell}^{2} A_{\ell}$. For an oblate spheroid $\left(a_{1}=a_{2}>a_{3}\right)$ with eccentricity $e=\sqrt{1-a_{3}^{2} / a_{1}^{2}}$, the "index symbols" (Chandrasekhar 1969) are

$$
\begin{gathered}
A_{1}=A_{2}=\frac{\sqrt{1-e^{2}}}{e^{3}} \arcsin (e)-\frac{1-e^{2}}{e^{2}} . \\
A_{3}=\frac{2}{e^{2}}-\frac{2 \sqrt{1-e^{2}}}{e^{3}} \arcsin (e) .
\end{gathered}
$$

For points interior to the ellipsoid's surface, $U_{\text {surf }}=-\mathfrak{V}$.

\subsubsection{Surface points}

The gravitational potential at an ellipsoid's surface is

$U_{\text {surf }}=-\pi G d\left(\left(A_{1} \frac{a^{2}}{c^{2}}+A_{3}\right) c^{2}+\left(A_{1} \frac{a^{2}}{c^{2}}-A_{3}\right) z^{2}\right)$.

\subsubsection{Points exterior to the suface}

At an exterior point $\boldsymbol{r}=\left(x_{1}, x_{2}, x_{3}\right)$, the potential energy (Chandrasekhar 1969) is

$$
\mathfrak{V}=\pi G d a_{1} a_{2} a_{3} \int_{\lambda}^{\infty} \frac{\mathrm{d} u}{\Delta}\left(1-\sum_{i=1}^{3} \frac{x_{i}^{2}}{a_{i}^{2}+u}\right),
$$

where $\Delta=\sqrt{\left(a_{1}^{2}+u\right)\left(a_{2}^{2}+u\right)\left(a_{3}^{2}+u\right)}$. The lower limit $\lambda$ of integration is determined implicitly by the equation

$$
\sum_{i=1}^{3} \frac{x_{i}^{2}}{a_{i}^{2}+\lambda}=1
$$

For points exterior to the ellipsoid's surface, $U_{\text {surf }}=-\mathfrak{V}$.

For oblate spheroids, the above integrals may be evaluated as follows:

$$
\begin{aligned}
\int_{\lambda}^{\infty} \frac{\mathrm{d} u}{\Delta} & =\frac{\pi-2 \operatorname{arccot}\left(e / \sqrt{\lambda / a_{1}^{2}+1-e^{2}}\right)}{a_{1} e} \\
\lim _{e \rightarrow 0^{+}} \int_{\lambda}^{\infty} \frac{\mathrm{d} u}{\Delta} & =\frac{2}{a_{1} \sqrt{\lambda / a_{1}^{2}+1}} \\
\int_{\lambda}^{\infty} \frac{\mathrm{d} u}{\Delta\left(a_{1}^{2}+u\right)} & =\frac{\left(\pi-2 \operatorname{arccot}\left(e / \sqrt{\lambda / a_{1}^{2}+1-e^{2}}\right)\right)}{2 a_{1}^{3} e^{3}} \\
\lim _{e \rightarrow 0^{+}} \int_{\lambda}^{\infty} \frac{\mathrm{d} u}{\Delta\left(a_{1}^{2}+u\right)} & =\frac{-\frac{\sqrt{\lambda / a_{1}^{2}+1-e^{2}}}{a_{1}^{3} e^{2}\left(\lambda / a_{1}^{2}+1\right)}}{3 a_{1}^{3}\left(\lambda / a_{1}^{2}+1\right)^{\frac{3}{2}}}, \\
\int_{\lambda}^{\infty} \frac{\mathrm{d} u}{\Delta\left(a_{3}^{2}+u\right)}= & -\frac{\left(\pi-2 \operatorname{arccot}\left(e / \sqrt{\lambda / a_{1}^{2}+1-e^{2}}\right)\right)}{a_{1}^{3} e^{3}} \\
\lim _{e \rightarrow 0^{+}} \int_{\lambda}^{\infty} \frac{\mathrm{d} u}{\Delta\left(a_{3}^{2}+u\right)}= & +\frac{2}{a_{1}^{3} e^{2} \sqrt{\lambda / a_{1}^{2}+1-e^{2}}} \\
& =\frac{2}{a_{1}^{3}\left(\lambda / a_{1}^{2}+1\right)^{\frac{3}{2}}}
\end{aligned}
$$

\subsection{Potential energy due to the external toroidal mass}

For a mass $M_{\text {ext }}$ distributed uniformly along a circle of radius $R_{0}$ in the $z=0$ plane external to the ellpsoid with linear mass density $M_{\text {ext }} / 2 \pi R_{0}$, the gravitational potential energy at a point $r$ is

$U_{\text {ext }}(\boldsymbol{r})=-\frac{G M_{\mathrm{ext}}}{2 \pi R_{0}} \int_{0}^{2 \pi} \frac{R_{0} \mathrm{~d} \theta^{\prime}}{\left|\boldsymbol{r}-\boldsymbol{r}^{\prime}\right|}$,

where the integration is over the circumference of a circle of radius $R_{0}$, the points of which are denoted by $\boldsymbol{r}^{\prime}$. In cylindrical coordinates $\boldsymbol{r}=(r, \theta, z), \boldsymbol{r}^{\prime}=\left(R_{0}, \theta^{\prime}, 0\right)$ and the Green's function is

$\frac{1}{\left|\boldsymbol{r}-\boldsymbol{r}^{\prime}\right|}=\frac{1}{\pi \sqrt{r R_{0}}} \sum_{m=-\infty}^{\infty} \mathrm{e}^{\mathrm{i} m\left(\theta-\theta^{\prime}\right)} Q_{m-1 / 2}(\chi)$, 
where

$\chi=\frac{\left(r^{2}+R_{0}^{2}+z^{2}\right)}{2 r R_{0}}$

and $Q_{m-1 / 2}$ is the half-integer Legendre function of the second kind (Cohl \& Tohline 1999).

The integrals for every term in the summation except $m=0$ are zero and the potential simplifies to

$U_{\mathrm{ext}}(\boldsymbol{r})=-\frac{G M_{\mathrm{ext}}}{\pi \sqrt{r R_{0}}} Q_{-1 / 2}(\chi)$.

The $m=0$ Legendre function of the second kind is $Q_{-1 / 2}(\chi)=\mu K(\mu)$, where $K$ is the complete elliptic integral of the first kind and

$\mu=\sqrt{\frac{2}{1+\chi}}=\sqrt{\frac{4 r R_{0}}{\left(r+R_{0}\right)^{2}+z^{2}}}$.

Therefore the potential field due to the external mass is

$U_{\mathrm{ext}}(r, z)=-\frac{2 G M_{\mathrm{ext}}}{\pi \sqrt{\left(r+R_{0}\right)^{2}+z^{2}}} K(\mu)$.

A useful approximation to the complete elliptic integral of the first kind is

$$
\begin{aligned}
K(\mu) & \approx \frac{\pi}{2(1-\mu)^{1 / 4}} \\
& \approx \frac{\pi}{2}\left(1+\frac{\mu}{4}+\frac{9 \mu^{2}}{64}+\frac{25 \mu^{3}}{256}+\frac{1225 \mu^{4}}{16384}+\cdots\right) .
\end{aligned}
$$

For large $R_{0}$ the potential field may be approximated by a Taylor series in $1 / \sqrt{R_{0}}$, we obtain

$$
\begin{aligned}
U_{\text {ext }}(r, z)= & -G M_{\text {ext }}\left(\frac{1}{R_{0}}+\frac{1}{2} \sqrt{r} \frac{1}{R_{0}^{3 / 2}}-\frac{7}{16} r \frac{1}{R_{0}^{2}}\right) \\
& +G M_{\text {ext }}\left(\frac{7}{32} r^{3 / 2} \frac{1}{R_{0}^{5 / 2}}+\frac{512 z^{2}-521 r^{2}}{1024} \frac{1}{R_{0}^{3}}+\cdots\right) .
\end{aligned}
$$

\subsection{Interaction energy between the Maclaurin spheroid and the external toroidal mass}

The gravitational interaction energy between a spheroid and the toroidal mass is

$$
\begin{aligned}
U_{\text {sph-tor }} & =\int U_{\text {ext }}(\boldsymbol{r}) \mathrm{d}(\boldsymbol{r}) \mathrm{d}^{3} r \\
& =\mathrm{d} \int_{0}^{a} 2 \pi r \mathrm{~d} r \cdot 2 \int_{0}^{c \sqrt{1-r^{2} / a^{2}}} \mathrm{~d} z[F(r, z)] \\
& =-\frac{3 G M^{2}}{R^{3}}\left(\frac{M_{\text {ext }}}{M}\right) \int_{0}^{a} r \mathrm{~d} r[G(r, u)],
\end{aligned}
$$

where

$F(r, z)=-\frac{2 G M_{\mathrm{ext}}}{\pi \sqrt{\left(r+R_{0}\right)^{2}+z^{2}}} K(\mu)$,

$G(r, u)=\sinh ^{-1}(u)+\frac{\sqrt{r R_{0}}}{2} \frac{\tan ^{-1}(u)}{R_{0}+r}+\cdots$,

and $u=c \sqrt{1-r^{2} / a^{2}} /\left(R_{0}+r\right)$.
When $R_{0}$ is large compared to the dimensions of the ellipsoid, the interaction energy $U_{\text {st }} \equiv U_{\text {sph-tor }} / U_{0}$ reduces to

$$
\begin{aligned}
U_{\mathrm{st}}= & -\frac{G M M_{\mathrm{ext}}}{R_{0} U_{0}}-0.6243 \gamma^{-1 / 12}\left(\frac{M_{\mathrm{ext}}}{M}\right)\left(\frac{R}{R_{0}}\right)^{3 / 2} \\
& +\frac{5 \pi^{2}}{16} \gamma^{-1 / 6}\left(\frac{M_{\mathrm{ext}}}{M}\right)\left(\frac{R}{R_{0}}\right)^{2}+O\left(\left(\frac{R}{R_{0}}\right)^{5 / 2}\right) \\
= & -\frac{G M M_{\mathrm{ext}}}{R_{0} U_{0}}-\eta \gamma^{-1 / 12}+\cdots,
\end{aligned}
$$

where $\gamma=\left(1-e^{2}\right)$ and the small perturbation parameter for the external mass is

$\eta=0.6243\left(\frac{M_{\mathrm{ext}}}{M}\right)\left(\frac{R}{R_{0}}\right)^{3 / 2}$

\subsection{Interaction energy between the "steep profile" and the external toroidal mass}

Using the Taylor series for the gravitational potential, Eq. (63), the interaction energy $U_{\text {sph-tor }}$ between a "steep profile" and the toroidal mass is

$$
\begin{aligned}
& U_{\text {sph-tor }}=d \int \mathrm{d} z \int_{0}^{r_{\epsilon \lambda}(z)} 2 \pi r \mathrm{~d} r U_{\text {ext }}(\boldsymbol{r}) \\
& =-\frac{G M M_{\mathrm{ext}}}{R_{0}}-\frac{\pi \mathrm{d} G M_{\mathrm{ext}}}{2 R_{0}^{3 / 2}} \int_{0}^{\infty} \mathrm{d} z \int_{0}^{r_{\epsilon \lambda}} r^{3 / 2} \mathrm{~d} r+O\left(R_{0}^{-2}\right) \\
& =-\frac{G M M_{\mathrm{ext}}}{R_{0}}-U_{0} \frac{M_{\mathrm{ext}}}{M}\left(\frac{R}{R_{0}}\right)^{3 / 2} 2^{7 / 6} \int_{0}^{\infty} r_{\epsilon \lambda}^{5 / 2}(z) \mathrm{d} z+\cdots \\
& \approx-\frac{G M M_{\mathrm{ext}}}{R_{0}}-U_{0} \frac{M_{\mathrm{ext}}}{M}\left(\frac{R}{R_{0}}\right)^{3 / 2} 2^{7 / 6} \lambda^{7 / 2} \\
& \times \int_{0}^{\infty}\left[r_{\mathrm{sph}}^{5 / 2}(u)+\frac{5}{4} \epsilon r_{\mathrm{sph}}^{1 / 2}(u) \Delta g(u)\right] \mathrm{d} u \\
& \approx-\frac{G M M_{\mathrm{ext}}}{R_{0}}-U_{0} \frac{M_{\mathrm{ext}}}{M}\left(\frac{R}{R_{0}}\right)^{3 / 2} 2^{1 / 6}(1+0.03703 \epsilon) \\
& \times\left[2 \int_{0}^{1 / 2}\left(1-4 z^{2}\right)^{5 / 4} \mathrm{~d} z+\frac{5 \epsilon}{2} \int_{0}^{1 / 2}\left(1-4 z^{2}\right)^{1 / 4} \Delta g \mathrm{~d} z\right] \\
& =-\frac{G M M_{\mathrm{ext}}}{R_{0}}-U_{0} \frac{M_{\mathrm{ext}}}{M}\left(\frac{R}{R_{0}}\right)^{3 / 2} 2^{1 / 6}(1+0.03703 \epsilon) \\
& \times(0.6243-0.02824 \epsilon) \\
& =-\frac{G M M_{\mathrm{ext}}}{R_{0}}-\eta U_{0} 2^{1 / 6}(1+0.03703 \epsilon)(1-0.04523 \epsilon) \\
& =U_{\mathrm{s}-\mathrm{t}}+(0.00921) \eta U_{0} \epsilon \text {. }
\end{aligned}
$$

\subsection{Equilibrium configurations of the rotating masses}

By minimizing the total energy, we determine equilibrium configurations.

\subsubsection{Total energy of the Maclaurin spheroid}

The total energy $E$ of an ellipsoid of eccentricity $e$ is the sum of the kinetic energy of rigid rotation and the gravitational selfenergy,

$E=\frac{1}{2} \lambda^{2} \gamma^{1 / 3}-\gamma^{1 / 6} \frac{\arcsin (e)}{e}$, 
where the energies are in units of $U_{0}$ and the angular momentum $\lambda$ is in units of $\sqrt{\mathcal{I}_{0} U_{0}}$. Minimization of the energy determines the Maclaurin spheroid expression for the angular momentum as a function of the eccentricity,

$\lambda_{\text {Maclaurin }}^{2}=\frac{1}{e^{2} \gamma^{1 / 6}}\left[\left(3-2 e^{2}\right) \frac{\arcsin (e)}{e}-3 \sqrt{\gamma}\right]$.

\subsubsection{Total energy of the Maclaurin spheroid + external toroidal mass}

The total energy is

$E=\frac{1}{2} \lambda^{2} \gamma^{1 / 3}-\gamma^{1 / 6} \frac{\arcsin (e)}{e}-\eta \gamma^{-1 / 12}$.

Energy minimization determines the angular momentum of the equilibrium states

$\lambda^{2}=\lambda_{\text {Maclaurin }}^{2}-\frac{\eta}{2 \gamma^{5 / 12}}$.

Note that the zero angular momentum equilibrium solution occurs at eccentricity $e>0$.

\subsubsection{Total energy of the "steep profile" + external toroidal mass}

When the energy is minimized for an ellipsoid with eccentricity $e=\sqrt{3} / 2$ plus an external toroidal mass, Eq. (73) determines the angular momentum. If the ellipsoid is deformed continuously into the "steep profile" while the angular momentum is held constant, the total energy changes from its spheroidal value $E_{\text {Maclaurin }}$ to

$E_{\text {"steep profile" }}=E_{\text {Maclaurin }}-(0.00005-0.0057 \eta) U_{0} \epsilon+\cdots$

This expression is a Taylor series which, for small $\eta$, determines accurately the derivative of the energy of the "steep profile" as a function of $\epsilon$. For large values of $\eta$, which is a measure of the strength of the interaction between the mass $M$ and the external toroidal mass $M_{\text {ext }}$, terms beyond the first two in the expansion of Eq. (63) must be included in the derivation. The "steep profile" has a lower energy than the spheroid when $\eta<0.008$ transforming the disky elliptical galaxy into a lenticular galaxy. The negative change from the spheroidal energy is the result of an increase in the kinetic energy that is offset by a slightly greater decrease in the gravitational self-energy.

\section{Discussion}

Our results demonstrate that the minor axes of both the isodensity surfaces of elliptical galaxies with aspect ratios $\leq 0.6$ and Maclaurin spheroidal masses will increase and morphologically form "steep profiles" when subjected to a small increase in their major axes in response to the gravitational force of a circumferential equatorial disk. The small displacement and consequently minimal force required to make this change may explain the transformation of a disky elliptical galaxy into a lenticular galaxy.

Disky elliptical galaxies can form as a consequence of the collapse of an elliptical galaxy. During the collapse sufficient outward transfer of angular momentum is generated to produce a circumferential equatorial disk (Larson 1975). Alternatively, disky elliptical galaxies may form from minor

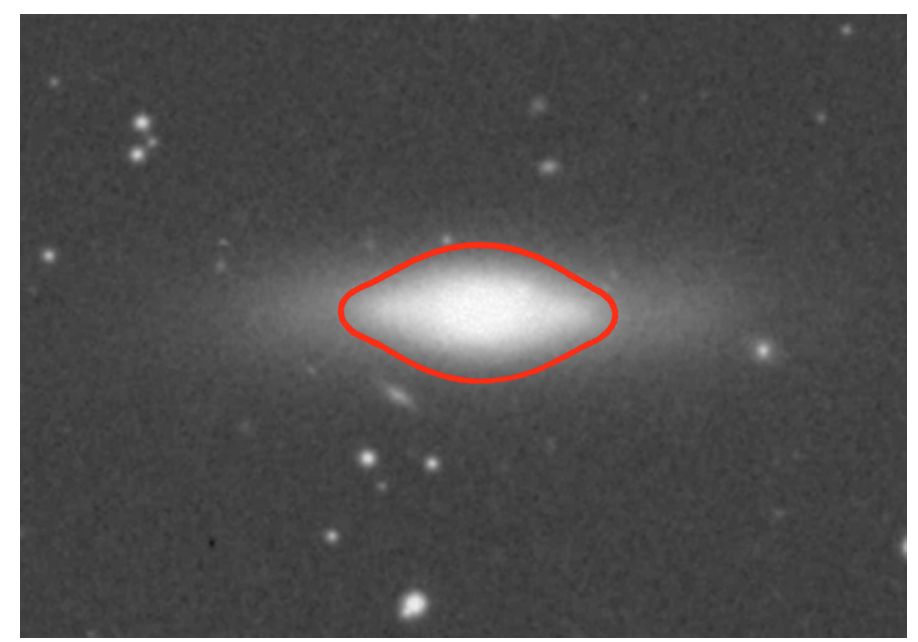

Fig. 10. A nearly edge-on photograph of lenticular galaxy NGC 2549 (Courtesy of Sloan Digital Sky Survey) with superposition of an oil film's "steep profile" outline on the lenticular galaxy's bulge.

mergers (Bournaud et al. 2007, Naab et al. 1999) or rotation (Jeans 1928). Disky elliptical galaxies appear to represent a different track for galaxy evolution (Bender et al. 1989, Nieto \& Bender, 1989, Kormendy et al. 2009).

Since such a disky elliptical galaxy rotates rapidly (Cappellari et al.2006 2007), its eccentricity will increase until it becomes an $\mathrm{E} 4$, aspect ratio $=0.6$. At that point, a small gravitational force generated by the circumferential equatorial disk will increase both the major and minor axes of the elliptical galaxy transforming it into a lenticular galaxy (Fig. 10). This process is more efficient as the aspect ratio of the disky elliptical galaxy declines below 0.6 (Schachar et al. 2008). This may explain why elliptical galaxies are $\leq \mathrm{E} 7$ and the majority are $\leq \mathrm{E} 4$ (Cresci et al.2006, Hao et al.2006).

According to the above discussion, the transformation of a disky elliptical galaxy into a lenticular galaxy seems to be a plausible process. Morphological high-resolution, multiwavelength data from high-resolution Hubble Space Telescope (HST) Advanced Camera Surveys suggests lenticular galaxy formation is a slow process (Wilman et al. 2009). The Sloan Digital Sky Survey (SDSS) shows that disky elliptical and lenticular galaxies are mostly found in isolated high density environments (Bamford et al. 2009). Lenticular galaxies exist where the gas density and temperature are too low for them to form by ram pressure striping or evaporation of a spiral galaxy and the bulgedisc ratio of lenticular galaxies is systematically larger than those of spiral galaxies (Dressler 1980). The distance between lenticular, elliptical and red spiral galaxies (Bamford et al. 2009) is smaller than the distance between lenticular and bluer spiral galaxies (Dressler et al.1997, Bamford et al. 2009).

The age of the bulge and the disc of lenticular galaxies (Fisher et al. 1996), kinematics, and properties of lenticular and elliptical galaxies are similar (Sandage \& Visvanathan 1978, Fisher et al. 1996, Jørgensen et al. 1996. De Propris et al. 2005. Cappellari et al. 2006. 2007. Emsellem et al. 2007a, Helmboldt 2007, Naab \& Ostriker 2009); however, it is important to appreciate and differentiate between slowly rotating elliptical galaxies, which have low ellipticities, are generally massive, metal-rich, and have shallow photometric profiles, and the rapidly rotating elliptical, disky elliptical and lenticular galaxies, which have high ellipticities, tend to be rotationally supported, are fainter 
objects, and tend to be metal-poor (Davies \& Illingworth 1983 , Jaffe et al. 1994, Kormendy \& Bender 1996, Erwin et al. 2004, Bedregal et al. 2007, Cappellari et al. 2006 2007). The distribution of fast and slow rotating galaxies and the evidence that boxy and disky ellipticals represent different evolutionary paths justifies separating fast and slow rotating galaxies (Bender et al. 1989. Nieto \& Bender 1989, Emsellem et al. 2007a. Kormendy et al.2009). Slowly rotating galaxies are more commonly classified as massive elliptical galaxies, while fast rotators are generally fainter and classified as elliptical or lenticular galaxies (Bender 1988, Emsellem et al.2007b).

Francis \& Anderson (2009) have proposed a new theory for the formation of galaxy spiral arms that does not require a significant increase in galaxy rotational velocity. According to this theory, spiral arms are formed as a consequence of an increase in galaxy equatorial diameter. Once the spiral arms form they enlarge and eventually form two enduring spiral arms. This theory fits with our postulation of the transformation of a lenticular galaxy into a red spiral galaxy.

A small equatorial diameter expansion of a disky E4 in response to the gravitational force of its circumferential equatorial disk transforms the disky E4 into a lenticular galaxy. If the mass of the circumferential equatorial disk increases by accretion or a minor merger, it will apply more gravitational force, which will further expand the equatorial diameter of the lenticular galaxy initiating the formation of spiral arms as proposed by Francis \& Anderson (2009). In addition, lenticular galaxies have a range of rotational velocities (Emsellem et al. 2004, 2007a). Any increase in rotational velocity will enhance spiral arm formation (Jeans 1928) and the transformation of a lenticular galaxy into a red spiral galaxy.

The transition of a disky elliptical galaxy into a lenticular galaxy and then into a red spiral galaxy is consistent with their location with respect to the galaxies' group center. A greater percentage of elliptical galaxies are located closer to the group center than red spiral galaxies, which have a clear fractional peak that is located further from the group center. Approximately $30 \%$ of red spiral galaxies are located at $\sim 0.4 R_{\text {vir }}$. At higher densities or in the cores of groups the fraction of red spiral galaxies declines sharply (Bamford et al. 2009). This distribution of galaxy type in relationship to the group center suggests a timeline that is consistent with the proposed evolutionary path for red spiral galaxies. In contrast, blue spiral galaxies preferentially inhabit low density environments and they diminish steadily from the least dense to the most dense environments (Bamford et al,2009. Schawinski et al. 2009).

The proposed evolutionary pathway is opposite to the generally accepted formation of lenticular and elliptical galaxies from the merger of spiral galaxies. Although it is documented that galaxy mergers occur, it is our contention that this is not the major evolutionary path for lenticular or red spiral galaxies. That disky elliptical galaxies transform into lenticular galaxies which convert into spiral galaxies implies that the etiology of Hubble's "tuning fork" scheme should be re-examined. Jeans (1928) theorized that the Hubble's "tuning fork" was a consequence of galaxy rotation. As the rotational velocity of an elliptical galaxy increased it would progressively flatten and eventually form spiral arms. According to Jeans (1928) hypothesis rotational velocity should predict galaxy type and lenticular galaxies should have a smaller aspect ratio than their immediate elliptical predecessors. Neither of these predictions is true. Jeans (1928) was not aware of the existence of disky elliptical galaxies. Therefore he did not consider the implications of the gravitational force of the circumferential equatorial disk on the shape of a galaxy and its ability to initiate the formation of spiral arms with a minimal change in rotational velocity.

Although we contend that blue spiral galaxies follow a different evolutionary path than red spiral galaxies, it is possible that they are part of Hubble's "tuning fork" scheme. An E4 disky elliptical transforms into a lenticular galaxy that develops spiral arms converting it into a red spiral galaxy. The formation of spiral arms causes greater turbulence and pockets of high density generating molecular gas clouds from which protostars form (Francis \& Anderson 2009) transforming the red spiral galaxy into a blue spiral galaxy (Kannappan et al. 2009).

\section{Conclusions}

The main result of this paper is our novel explanation for the observation that the aspect ratio of lenticular galaxies is greater than their immediate elliptical predecessors. The idea is that a small increase in the major axis of an oblate object with an aspect ratio $\leq 0.6$ causes its minor axis to increase (Schachar et al. 2008). The circumferential equatorial disk of an E4 elliptical galaxy supplies the gravitational force required to increase its equatorial diameter. This causes the E4 to transform into a lenticular galaxy. An increase in mass of the disk of the lenticular galaxy causes the equatorial diameter of the lenticular galaxy to expand initiating the formation of spiral arms (Francis \& Anderson 2009). This converts the lenticular galaxy into a red spiral galaxy. The common environment of disky elliptical, lenticular, and red spiral galaxies, and their fractional groupocentric distances support this evolutionary process (Bamford et al.2009).

Acknowledgements. We thank the anonymous referee for a careful reading of the manuscript and the invaluable, thoughtful suggestions that significantly improved and broadened the implications of our work.

\section{References}

Abraham, R. G., \& van den Bergh, S. 2001, Sci, 293, 1273

Baes, M. 2008, MNRAS, arxiv.org/pdf/0810.5483v1

Bamford, S. P., Nichol, R. C., Baldry, I. K., et al. 2009, MNRAS, 393, 1324

Bedregal, A. G., Aragón-Salamanca, A. Merrifield, M. R., \& Milvang-Jensen, B. 2006, MNRAS, 371, 1912

Bedregal, A. G., Aragón-Salamanca, A., Merrifield, M. R., \& Cardiel, N. 2007, in Proc. IAU Symp., 241, 1

Bender, R. 1988, A\&A, 193, L7

Bender, R., Surma, P., Döbereiner, S., et al. 1989, A\&A 217, 35

Bournaud, F., Jog, C. J., \& Combes, F. 2007, A\&A, 476, 1179

Caon, N., \& Einasto, M. 1995, MNRAS, 273, 913

Cappellari, M. 2008, MNRAS, 390, 71

Cappellari, M. Bacon, R., Bureau, M., et al. 2006, MNRAS, 366, 1126

Cappellari, M., Emsellem, E., Bacon, R., et al. 2007, MNRAS, 379, 418

Chandrasekhar, S. 1969, Ellipsoidal Figures of Equilibrium (New Haven: Yale Univ. Press)

Cohl, H. S., Tohline, \& J. E. 1999, ApJ, 527, 86

Cresci, G., Davies, R. I., Baker, A. J., et al. 2006, A\&A, 458, 385

Dankova, Ts., \& Rosensteel, G. 1998, Am. J. Phys. 66, 1095

Davies, R. L., \& Illingworth, G. 1983, ApJ, 266, 516

De Propris, R., Colless, M., Driver, S. P., et al. 2005, MNRAS, 357, 590

Dressler A. 1980, ApJ, 236, 351

Dressler, A., Oemler Jr., A., Couch, W. J., et al. 1997, ApJ, 490, 577

Emsellem, E., Cappellari, M., Peletier, R. F., et al. 2004, MNRAS, 352, 721

Emsellem, E., Cappellari, M., Krajnović, D., et al. 2007a, MNRAS, 379, 401

Emsellem, E., Cappellari, M., Krajnović, D., et al. 2007b, Proc. IAU Symp.,

245,1

Eriguchi, Y., \& Hachisu, I. 1982, Prog. Theor. Phys., 67, 844

Erwin, P., Beckman, J. E., \& Beltrán, J. C. V. 2004,

arxiv.org/pdf/0409103v1

Fisher, D., Franx, M., \& Illingworth, G. 1996, ApJ, 459, 110

Francis, C., \& Anderson, E. 2009, arxiv.org/pdf/0901.3503v2

Hao, C. N., Mao, S., Deng, Z. G., et al. 2006, MNRAS, 370, 1339 
Helmboldt, J. F. 2007, MNRAS 379, 1227

Hernandez, X., \& Lee, W. H. 2004, MNRAS, 347, 1304

Hubble, E., 1926, AJ, 64, 321

Hubble, E. P. 1936, The Realm of the Nebulae (New Haven: Yale Univ. Press)

Jaffe, W., Ford, H. C., O'Connell, R. W., et al. 1994, AJ, 108, 1567

Jeans, J. H. 1928, Astronomy and Cosmogony (Cambridge: Cambridge Univ. Press)

Jørgensen, I., \& Franx, M. 1994, ApJ, 433, 553

Jørgensen, I., Franx, M., \& Kjærgaard P. 1996, MNRAS, 280, 167

Kannappan, S. J., Guie, J. M., \& Baker, A. J. 2009, AJ, 138, 579

Katsiyannis, A. C., Boumis, P., \& Meaburn, J. 2001,

arxiv.org/pdf/0111443v1

Kormendy, J., \& Bender, R. 1996, ApJ, 464, L119

Kormendy, J., Fisher, D. B., Cornell, M. E., \& Bender, R. 2009, ApJ, Suppl. Ser., 182,216

Krajnović, D., Bacon, R., Cappellari, M., et al. 2008, MNRAS, 390, 93

Larson, R. B. 1975, MNRAS, 173, 671

Magorrian, J. 1999, MNRAS, 302, 530
Maller, A. H., Katz, N., Kereš, D., et al. 2006, ApJ, 647, 763

Michard, R., \& Marchal, J. 1994, Astron. Astrophys. Suppl. Ser. 105, 481

Miyamoto, M., \& Nagai, R. 1975, Publ. Astron. Soc. Japan, 27, 533

Naab, T., \& Ostriker J. P. 2009, ApJ 690, 1452

Naab, T., \& Burkert, A., \& Hernquist, L. 1999, ApJ, 523, L133

Nieto, J.-L., \& Bender, R. 1989, A\&A, 215, 266

Rix, H.-W., \& White, S. D. M. 1990, ApJ, 362, 52

Sandage, A. 2005, ARA\&A, 43, 581

Sandage, A., \& Visvanathan, N. 1978, ApJ, 225, 742

Satoh, C. 1980, Publ. Astron. Soc. Japan, 32, 41

Schachar, R. A., Liao, G. G., Kirby, R. D., et al. 2008, J. Phys. A: Math. Theor. 41,495204

Schawinski, K., Lintott, C., Thomas, D. et al. 2009, MNRAS,

arxiv.org/pdf/0903.3415v1

van den Bergh, S. 1980, Phil. Trans. R. Soc. Lond. A, 296, 319

Wilman, D. J., Oemler Jr., A., Mulchaey, J. S., et al. 2009, ApJ, 692, 298

Zhenglu, J. 2000, MNRAS, 319, 1067 